\title{
NETWORK OPERATORS ADVICE AND ASSISTANCE (NOAA): A REAL-TIME TRAFFIC REROUTING EXPERT SYSTEM
}

\author{
Rodney M. Goodman*, Barry Ambrose*, Hayes Latin** and Sandee Finnell ${ }^{* * *}$ \\ ${ }^{*}$ California Institute of Technology, ${ }^{* *}$ Systems Technology Pacific Bell, ${ }^{* * *}$ Southern California Network \\ Management Center Pacific Bell
}

\begin{abstract}
A real-time autonomous expert system has been developed to carry out traffic management in the Southern Californian telephone network. The system has been working on live data since September 1991 and generates rerouting advice that agrees with that generated by the present network management procedures. A modular software design was adopted to allow for evolution. A graphics interface allows the user to easily navigate through the display of exception conditions and advice. Exceptions are shown highlighted on a map of Southern California. A severity measure is calculated for each exception and is used to prioritize the display of information.
\end{abstract}

\section{Introduction}

In September 1990, a project was started by CalTech and Pacific Bell to investigate the implementation of a real-time autonomous expert system which would aid network management of the Pacific Bell Southern Californian telephone network. The ultimate aim of the project was to produce and automatically implement recommendations for network controls. To do this, the knowledge in everyday use by the network management staff had to be written down, and coded for use in a computer system while retaining flexibility and openness to change.

The situations that the network management staff are exposed to are complex. Every day new problems arise. Examples are single random overloads of traffic on a given route, high volume phone-in conditions, disasters such as earthquakes, unusual calling patterns such as occur on Mother's Day, killer trunks and other events. The introduction of new facilities, such as advanced signalling systems, adds additional problems that have to be diagnosed.

To date, a number of software modules have been developed which use data gathered in real time from the network. The software can produce a graphic analysis of the network, describe how the network is functioning in terms of exception conditions and recommend controls. The controls are given in the format that the network manager is accustomed to. Each module has been extensively tested.

0-7803-0608-2/92/\$3.00@ 1992 IEEE
The rest of the paper gives a description of the Pacific Bell telephone network and the architecture of the Network Operators Advice and Assistance (NOAA) system. This is followed by the details of how NOAA works presented logically in the order (i) input information available to NOAA (ii) decision making and decision support for NOAA (iii) controls recommended by NOAA. The map based Graphics Interface developed for NOAA is described separately and finally an indication is given of future work.

\section{The Pacific Bell Network}

In order to gain some appreciation of the network management tasks, one must have a description of the network to be managed. The Pacific Bell Southern Californian telephone network provides service to at least 1 million subscribers and has a hierarchical structure. For administrative and legal purposes it is divided into Local Access and Transport Areas (LATAs) [BELL86a] and the network management center in Pasadena manages LATA's 5 and 6. Geographically LATA's 5 and 6 extend to San Diego in the south and include all the Los Angeles urban area.

The network is hierarchical. End offices are the exchanges that serve customers, and tandems are the exchanges used for traffic between end offices that are not directly connected [BELL86a]. There are 6 tandems to be managed and over 200 end offices.

There are two types of trunk groups. High usage trunk groups are dimensioned to be lossy, i.e. during the busy hour they are not guaranteed to have enough capacity to carry all offered traffic. Traffic will therefore overflow onto the Final trunk groups which are dimensioned to provide a good Grade of Service. In general there will be a final route between each end office and its parent tandem. It is these final routes that provide the backbone of the network. The final routes are therefore closely monitored by the network managers. 


\section{Architecture of NOAA}

The Architecture of NOAA is shown in Figure 1. The Pacific Bell network management system is called EADAS [BELL86b]. NOAA is connected over a 4800 baud data link and appears as an ordinary operators terminal to EADAS. NOAA then runs on a Sun workstation.

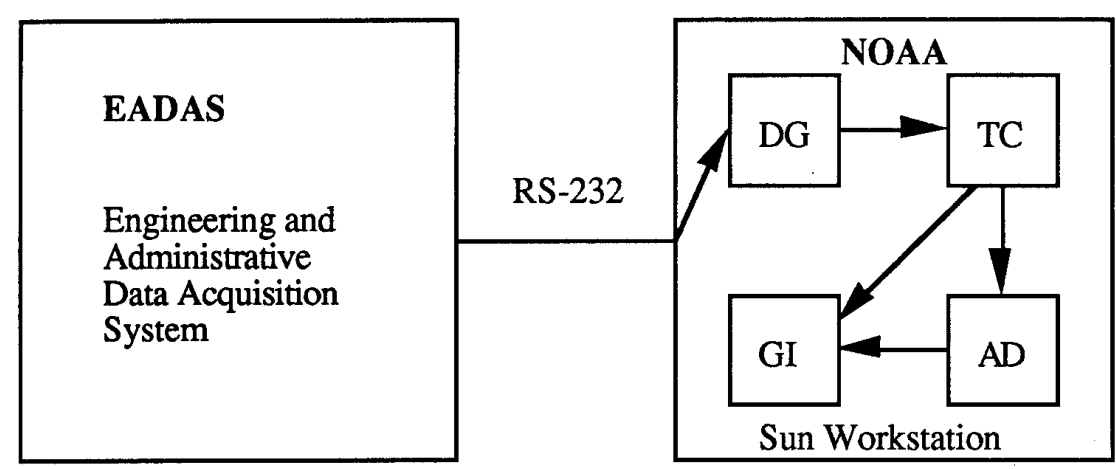

Figure 1 Architecture of NOAA

There are four software modules that make up NOAA, each made up of about 3000 lines of $C$ code. These are:

- $\quad$ data gathering (DG).

This module is responsible for logging into the EADAS system and maintaining the basic dialog with EADAS. The UNIX tip command is used for this purpose. A significant effort was put into making this module robust in the face of communication difficulties, such as loss of synchronization in the EADAS-NOAA dialogue.

- $\quad$ tandem calculations (TC).

This modules takes the data which has been downloaded from EADAS and carries out the calculations needed to transform the data into usable statistics. The data is downloaded in register format. These registers, also known as peg counts, are integer counts of events such as trunk seizures, that have happened in a five minute interval. The calculations that are carried out are well defined and produce more useful statistics such as seizures per circuit per hour.

\section{Advice ( $\mathrm{AD})$}

Given the statistics on the various trunk groups, exception situations are noted and a severity measure associated with each exception. A diagnosis based on observed symptoms is carried out according to rules supplied by network management staff. Finally advice is given about the response to the exception. If a control is advised, it is given in the format expected by the network operator. This can be then approved by the operator and implemented through EADAS.

- $\quad$ Graphics Interface (GI)

The graphics interface uses a map of Southern California. Exceptions are indicated by red, suggested controls by green and actual controls already in place by blue. Additional information is given when the operator clicks on the map. This information includes calculated statistics on the route and any advice generated for that route.

\section{Statistics}

NOAA makes its diagnosis on the basis of available information about the trunk groups. This information is available to NOAA in the form of counts of certain events for each trunk group during a 5 minute period. These events are for instance (i) a trunk being seized (ii) a seizure that is not successful i.e. overflow (iii) a survey of trunk occupancy at a given instant. On the basis of these counts, the following standard statistics are calculated:
$\mathrm{ACH}$
$\mathrm{CCH}$
Attempts per circuit per hour
OFL Connections per circuit per hour
USG Percentage of attempts that overflowed
HT Percentage of trunks occupied on average
HT Holding Time of Calls

The 5 minute period is a compromise. The shorter the period between data collections, the faster the response to any potential problems, but the larger the amount of information to be processed, requiring more expensive hardware. The 5 minute period means that problems can be cleared relatively rapidly. It is known that mean traffic increases do occur on a time scale of the holding time whereas traffic 
fluctuations occur on a time scale of the inter-arrival rate of calls [FILI90].

Since a large amount of information is available, filtering is carried out. Firstly exceptions on high usage routes are ignored and only final route data are examined. Secondly exceptions on special purpose final routes are ignored. For example, we expect a trunk group for the time announcement to have a short holding time. Thirdly overflows are treated as having higher priority than other exceptions, since an overflow on a final may represent lost traffic.

\section{Decisions}

When an exception condition has been noted on a trunk route, there could be many possible explanations for it. Typically phone-ins to radio stations and TV stations may generate excess call attempts. Facilities failures may mean that overflow shoots up on related trunk groups. Occasionally the data gathering may interfere with maintenance operations and unreliable data is returned. Finally random overflows can occur on individual trunk groups.

For any reroutes, a search for spare capacity is carried out. If there is an overflow problem between $A$ and $B$ where $A$ is an end office and B is a tandem, then the program first looks for capacity on other trunk groups going from A to B, and then carries out a search for $A$ to $B$ via $C$ possibilities. Here $C$ has to be one of the 6 tandem exchanges, because of the hierarchical nature of the network. The candidate reroutes are then sorted according to available capacity and controls are suggested that make use of the minimum number of reroutes that achieves the required capacity.

A severity measure is attached to each exception. This measure is biased to give overflow exceptions a high severity figure, since overflows can result in lost traffic. The severity measure is also made proportional to the size of the trunk group and the amount by which the statistic is above threshold. Thus a $10 \%$ overflow on 10 circuits is considered equivalent to $1 \%$ overflow on 100 circuits. A log scale is then used to scale the figure between 0 and 100 . This severity measure has proved useful for sorting exceptions prior to displaying them. In a full scale emergency with the loss of a lot of capacity, a sort by severity would indicate which trunk groups to deal with first. Note that 911 calls have their own reserved trunks from end offices and are not supervised by NOAA.
The rules used in the program are of three separate types:

- $\quad$ rules which indicate which exceptions can be safely ignored. For example overflow on high usage routes is ignored;

- $\quad$ rules which indicate which routes can be use as candidate reroutes;

- $\quad$ rules that map a suggested reroute into a list of controls to effect the reroutes. E.g. certain other routes may have to be "finalized" first to prevent a round-robin situation.

Some of the above rules were already written down in operators handbooks. Others were supplied by the network management staff. Examples of the rules are given in Table 1.

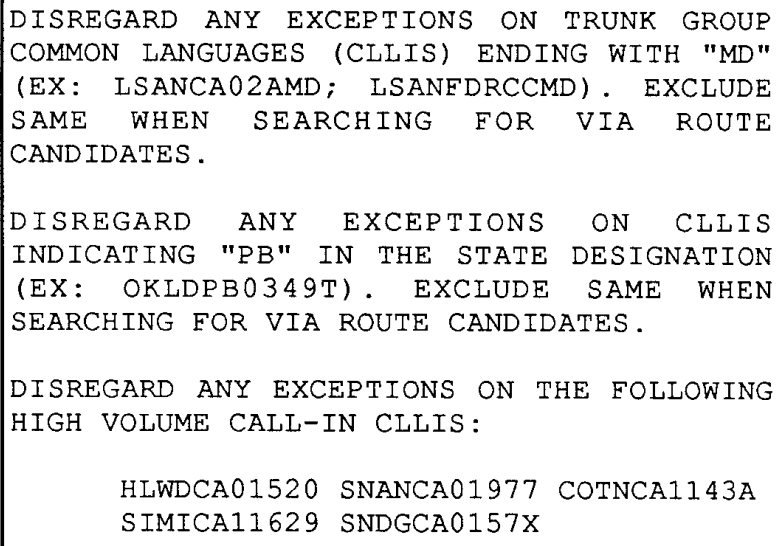

Table 1 Typical Network Management Rules

As with any rule based system, including the rules in a computer program has the advantage that any special cases are immediately recognized and the operators handbook does not have to be referred to. However for complete trust in the system, the rule base has to be extensively tested and compared with the experts analysis in a wide range of cases.

\section{Controls}

It was mentioned above that there are many causes of exceptions on the trunk groups. Similarly there are many possible controls that can be put in to improve the network throughput. In general, controls can be divided into protective controls and expansive controls [FILI90, BELL88]. 
Protective controls are used to cut down the amount of call attempts entering the network. For example in the event of a phone-in, controls can be put into each end office to block call-attempts to that telephone number. Alternatively a certain percentage can be let through. This limits the amount of congestion in the network. Clearly the calls destined for a phone-in are "bad" traffic as there is a high possibility of non completion, and therefore they should be blocked at source and not rerouted.

\section{Graphics Interface}

It was found that this application was eminently suitable for graphical interfacing. Initially a scrolling list of exception conditions was presented to the operator in a window. Buttons allowed the operator to pull up information about the statistics on a trunk group and the recommended advice.

This was then modified to allow the operator to choose from a number of lists of exceptions, each list corresponding to a different statistic over threshold.
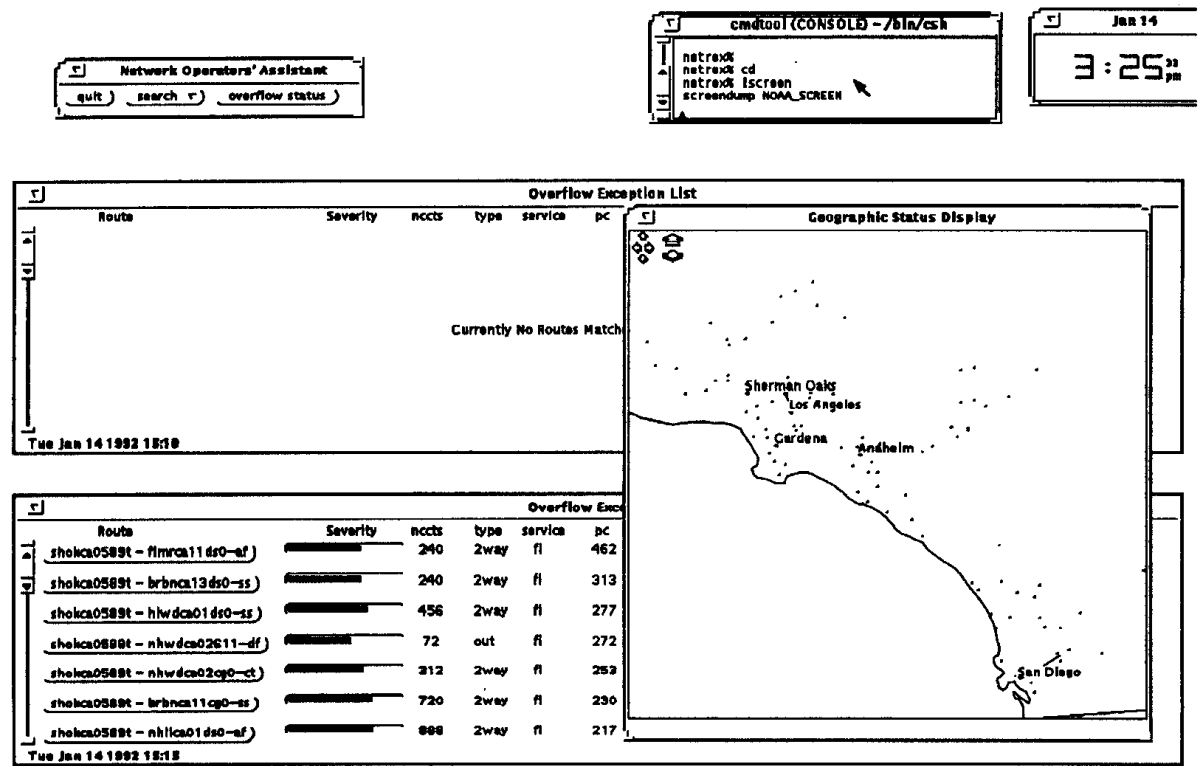

in in

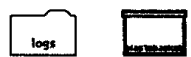

Figure 2 Graphical Interface for NOAA
Finally a map interface was developed (see Figure 2). Without doubt, this was the easiest way to (i) display an analysis of the network, showing the exceptions clearly (ii) pull up information about individual exceptions. The map, with its translation and zoom features, could be mastered by any user in minutes and added a "fun" element to navigation through the program. Coordinates of most of the cities in Southern California were available from a public database.

\section{Future Work}

On the other hand, expansive controls allow trunk groups that are normally separate to share their capacity. If there is a single random overload, then expansive controls are called for. Two alternatives here are overflow rerouting, which reroutes overflow traffic only, and an immediate reroute which puts all call attempts onto the alternative route first. Each is appropriate for certain cases. Finally spray rerouting is possible, whereby a number of alternatives are given and each is tried for a given call. Clearly todays telephone exchanges have a lot more flexibility than the hardwired mechanical exchanges that were common thirty years ago.
Over the past 18 months, much work has been done in interfacing NOAA to the Pacific Bell network management computer and building the infrastructure for an expert system. Over the coming months the rules implemented in the program will be tested by running the program on live data. When sufficient cases have been tested, it is intended to close the loop and allow NOAA to carry out controls autonomously. Clearly considerations of reliability and robustness have also to be taken into account when this step is to be carried out.

The ability of NOAA to diagnose problems correctly and to take the correct actions will be enhanced if the system has other information sources besides EADAS. Two other sources being considered at present are NSCS which provides information about 
seizures of trunks, and a separate system which provides information about the SS\#7 signalling network.

Looking at the long term future for NOAA, the definition of a standard data format for exceptions and for statistical information about trunk group performance would help in minimizing the cost of upgrade of NOAA, as new versions of EADAS become available. As in any network management application, standardization of data formats between applications that share the data is an important requirement. The ISO standards work on fault management and performance management should go some way to filling this gap.

To summarize, network management advice is currently being generated for the Southern California telephone network. As the rules that generate this advice are tuned, a robust network management application will have been developed that relieves network management staff of some of the need to supervise the day to day running of the telephone network.

\section{References}

BELL86a Bellcore, Network Management IntraLATA Network Fundamentals, BR 780150-122, Issue 1, December 1986.

BELL86b Bellcore, Network Management Technical Overview, BR 780-150-130, Issue 2, December 1986.

BELL88 Bellcore, Network Management Control Descriptions, BR 780-150-128, Issue 3, June 1988.

FILI90 Filipiak, J., Real Time Network Management, Draft Copy, April, 1990. 